\title{
THE ORDER OF DEGENERACY OF MARKOV CHAIN MONTE CARLO METHOD
}

\author{
Kengo Kamatani*
}

\begin{abstract}
Sometimes Markov chain Monte Carlo (MCMC) procedures work poorly. The identification of this inefficiency is important, but appropriate theoretical tools have not been investigated adequately. For this purpose, we propose the order of degeneracy, which measures the mixing property of an MCMC procedure. As an application, we consider major three sources of inefficiency, one being the fragility of the identification of parameters. We present a numerical simulation to show the effect of each source of inefficiency.
\end{abstract}

Key words and phrases: Bernstein-von Mises theorem, Markov chain, Monte Carlo method, probit model.

\section{Introduction}

The Markov chain Monte Carlo (MCMC) method is an efficient tool for the approximation of an integral with respect to a particular type of probability measure. The strategy dates back to Metropolis et al. (1953), Hastings (1970), and it was later implemented for Bayesian statistics (Tanner and Wong (1987), Gelfand and Smith (1990)). Since then, the MCMC method has been one of the most popular methods for the evaluation of complicated integrals with respect to the posterior distribution. For a recent review, see Robert and Casella (2011).

Let $P(d x \mid \theta)$ be a probability measure with prior distribution $P(d \theta)$. Sometimes the posterior distribution $P(d \theta \mid x)$ does not have a closed form and requires an approximation. In this paper, we focus mainly on the procedure referred as data augmentation (DA) procedure, which uses the so-called augmented-data model $P(d x, d y \mid \theta)$ that introduces a working variable $y$ such that $\int_{y} P(d x, d y \mid$ $\theta)=P(d x \mid \theta)$. The DA procedure is defined by the following iteration:

$$
\text { simulate } y|x, \theta \sim P(d y \mid x, \theta), \quad \theta| x, y \sim P(d \theta \mid x, y) \text {. }
$$

This procedure results in the Markov chain $\theta_{\infty}=\left(\theta_{m}\right)_{m \in \mathbb{N}_{0}}$ with the invariant distribution $P(d \theta \mid x)$ (see Tierney (1994), Gilks et al. (1996), Roberts and Rosenthal (2004)). Under mild conditions, the value of $\int \theta P(d \theta \mid x)$ is well approximated by $M^{-1} \sum_{m=0}^{M-1} \theta_{m}$. When the convergence is fast and $P(d \theta \mid x)$ is complicated, this strategy is very advantageous compared to other numerical integration methods.

Although many efforts have been made to analyze the convergence property of the MCMC (such as Roberts and Rosenthal (2004), Diaconis et al. (2008); also

\footnotetext{
Received September 4, 2013. Revised December 20, $2013 . \quad$ Accepted January 15, 2014.

*Graduate School of Engineering Science, Osaka University, 1-3 Machikaneyama-cho, Toyonaka-si, Osaka 560-0043, Japan.
} 
see Robert and Casella (2011)), theories on the efficiency of this approximation are being developed. The purpose of the present paper is to assist this effort and provide a measure of efficiency. To illustrate our approach, we consider the model (1.2) below:

$$
P(x=1 \mid \theta)=\Phi(\theta), \quad P(x=0 \mid \theta)=\Phi(-\theta) .
$$

Let $x_{n}=\left(x^{i}\right)_{i=1, \ldots, n}$ be an i.i.d. copy and let $N(0,1)$ be the prior distribution. Consider the following two choices of augmented-data model and construct the corresponding DA procedures:

$$
y \mid \theta \sim N(0,1) \quad \text { and } \quad x=1_{\{y \leq \theta\}}
$$

and

$$
y \mid \theta \sim N(-\theta, 1) \quad \text { and } \quad x=1_{\{y \leq 0\}} .
$$

Although the two DA procedures are similar and have geometric ergodicities, their performances are quite different (Figure 1).

Using existing techniques, such as comparing operator norms, it is not easy to perform a theoretical comparison of the two MCMC strategies. However, as the sample size $n \rightarrow \infty$ for the two DA procedures based on (1.3) and (1.4), $\mid \theta_{m+1}-$ $\theta_{m} \mid(m=0,1, \ldots)$ are on the order of $O_{P}\left(n^{-1}\right)$ and $O_{P}\left(n^{-1 / 2}\right)$, respectively. The former procedure does not converge as quickly as the latter and thus has a poorer performance.

With this property in mind, we propose the order of degeneracy; in an MCMC procedure, we specify the rate $d_{n}$ that determines the rate of $\left|\theta_{m+1}-\theta_{m}\right|$
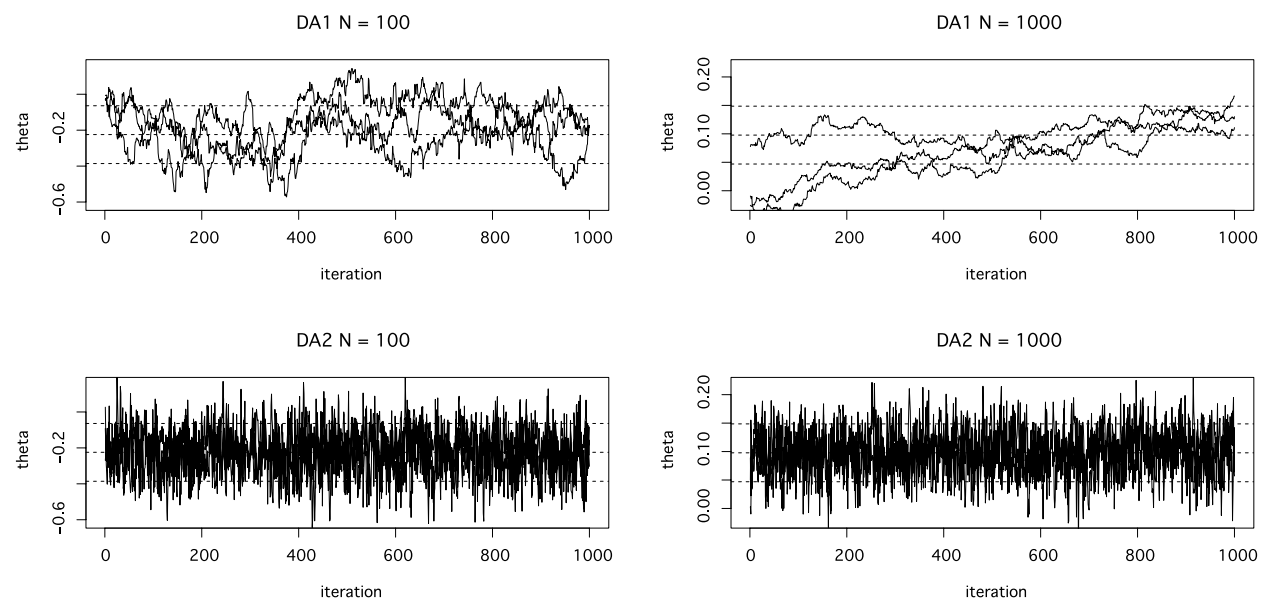

Figure 1. DA procedures for (1.3) (upper) and (1.4) (lower) with sample sizes $n=100$ (left) and $n=1000$ (right). Shown are trajectories $\theta_{0}, \theta_{1}, \ldots$ for three runs for each, for an observation $x_{n}=\left(x^{1}, \ldots, x^{n}\right)$. The dashed lines are the 10th, 50th, and 90th percentiles of the posterior distribution. 
Table 1. Order of degeneracy of the DA procedures for various conditions.

\begin{tabular}{clcc}
\hline Subsection & Source of inefficiency & Order of $\left|\theta_{1}-\theta_{0}\right|^{-1}$ & the order of degeneracy \\
\hline 3.1 & Variable dominance & $\epsilon^{-1} \sqrt{n}$ & $\epsilon^{-3 / 2}$ \\
3.2 & Fragility of identification & $\sqrt{n}$ & $\epsilon^{-1}$ \\
3.3 & Large information & $n$ & $\sqrt{n}$ \\
\hline
\end{tabular}

$(m=0,1, \ldots)$ and we can compare different MCMC procedures by this rate. This calculation is usually very easy. We apply order of degeneracy to three major sources of inefficiency. Some of our results are summarized in Table 1.

These rates come from the local behavior of MCMC procedures that is useful for the analysis of MCMC procedures (see Kamatani (2011, 2013a, 2014)). Without knowledge of the local properties, we may fail to detect inefficient use of MCMC procedures. For example, in Subsection 3.3, we will show that the usual autocorrelation plots cannot detect poor behavior of the PX-DA procedure, but the order of degeneracy can.

The rest of the paper is organized as follows. Section 2 reviews the necessary background. The order of degeneracy is also defined in this section. In Section 3, we apply the order of degeneracy to three models. Numerical simulations are presented in Section 4.

We write $X \mid Y$ for the probability law of $X$ conditioned on $Y$. We also write $X \mid Y \sim P(d x \mid Y)$ if the law of $X$ conditioned on $Y$ is $P(d x \mid Y)$. We assume that $P(d x \mid Y=y)$ is a probability measure for each $u$ and $y \mapsto P(A \mid Y=y)$ is measurable. We write $x_{n}=\left(x^{i}\right)_{i=1, \ldots, n}, y_{n}=\left(y^{i}\right)_{i=1, \ldots, n}$ and $z_{n}=\left(z^{i}\right)_{i=1, \ldots, n}$.

\section{Order of degeneracy}

\subsection{Local properties}

Let $\left(\Omega_{n}, \mathcal{F}_{n}, \mathbb{P}_{n}\right)$ be a sequence of probability spaces, and let $\Theta$ be an open subset of $\mathbb{R}^{d}$. We assume that

$$
x_{n}=\left(x^{i}\right)_{i=1, \ldots, n} \sim P_{n}\left(d x_{n}\right):=\int_{\Theta} \prod_{i=1}^{n} P\left(d x^{i} \mid \theta\right) P(d \theta),
$$

where $P(d x \mid \theta)$ is a parametric family, and $P(d \theta)$ is the prior distribution. Let $\theta_{\infty}=\left(\theta_{m}\right)_{m \in \mathbb{N}_{0}}$ be an MCMC output, which is an infinite vector of elements of $\Theta$. We assume that $\theta_{\infty} \mid x_{n}$ is a Markov chain that has the posterior $P\left(d \theta \mid x_{n}\right)$ as the invariant distribution. We also assume stationarity of $\theta_{\infty} \mid x_{n}$ throughout this paper, that is, the initial guess $\theta_{0} \mid x_{n}$ is generated from $P\left(d \theta \mid x_{n}\right)^{1}$. By classical results such as (3.6) of Doob (1949), the posterior distribution almost certainly (so-called consistency) tends to a point mass as the sample size $n$ grows. The order of this convergence is usually $\sqrt{n}$, but is sometimes $n^{1 / \alpha}$ or $(n \log n)^{1 / 2}$ (see Akahira and Takeuchi (1995)). In this paper, we write this order as $c_{n}$. More precisely, the order of consistency is defined as follows:

1 This assumption may seem to be strong in practice since usually the MCMC output $\theta_{\infty}$ is invariant to $P\left(d \theta \mid x_{n}\right)$ but is not a stationary process. However, this assumption can be weakened. See Lemma 4 of Kamatani (2014) 
DEFinition 2.1.1 (Order of consistency). Let $\theta$ and $\theta_{*}$ be independent draws from $P\left(d \theta \mid x_{n}\right)$. The increasing positive sequence $c_{n}$ such that

$$
c_{n}\left|\theta-\theta_{*}\right|=O_{\mathbb{P}_{n}}(1)
$$

is called the order of consistency.

The output $\theta_{\infty}$ is used to approximate integrals with respect to the posterior distribution. We expect the approximation to be accurate. To analyze the validity of the approximation, we compare the integral $I^{n}:=\int \psi\left(c_{n}\left(\theta-u_{n}\right)\right) P\left(d \theta \mid x_{n}\right)$ and the approximation $I_{M}^{n}:=M^{-1} \sum_{m=0}^{M-1} \psi\left(c_{n}\left(\theta_{m}-u_{n}\right)\right)$, where $u_{n}\left(x_{n}\right)$ is a particular statistic such as the maximum likelihood estimator and $\psi$ is a real-valued bounded continuous function. It would be helpful if, for any $M_{n} \rightarrow \infty$,

$$
I^{n}-I_{M_{n}}^{n}=O \mathbb{P}_{n}(1) .
$$

In Kamatani (2014), this property was called the local consistency of the MCMC procedure and was noted as a useful property. On the other hand, the local degeneracy of the MCMC procedure was defined in Kamatani (2011) as a property of inefficiency. The MCMC procedure has local degeneracy if, for any $M \in \mathbb{N}$ and for any continuous bounded function $\psi$,

$$
I_{1}^{n}-I_{M}^{n}=o \mathbb{P}_{n}(1)
$$

This means that the approximations to the posterior distribution obtained by $M$ iterations of the MCMC procedure are no more helpful than that using only one iteration.

The following proposition illustrates the relationship between these two properties. See Kamatani (2011) for its proof.

Proposition 2.1. Assume $c_{n}\left(\theta-u_{n}\right)=O_{\mathbb{P}_{n}}(1)$, where $\theta \sim P\left(d \theta \mid x_{n}\right)$. Suppose that $\theta_{\infty} \mid x_{n}$ is stationary with invariant probability measure $P\left(d \theta \mid x_{n}\right)$. Then

(i) Local degeneracy is equivalent to $c_{n}\left(\theta_{1}-\theta_{0}\right)=o \mathbb{P}_{n}(1)$.

(ii) If the MCMC procedure satisfies both local consistency and local degeneracy, then there exists a random variable $u_{n}^{\prime}\left(x_{n}\right)$ such that $c_{n}\left(\theta-u_{n}^{\prime}\right)=o \mathbb{P}_{n}(1)$.

By the above, no locally consistent MCMC can have local degeneracy if $c_{n}\left(\theta-u_{n}\right) \neq o \mathbb{P}_{n}(1)$ for any $u_{n}\left(x_{n}\right)$. This inefficiency property, local degeneracy, is somewhat too broad. Almost all the examples in this paper have local degeneracy, but they vary in their performance. The rate of convergence was considered in Kamatani (2013a) to be useful for the classification of MCMC procedures.

DEFinition 2.1.2. For a sequence of real numbers $r_{n}$, if (2.3) holds for any $M_{n}$ such that $M_{n} / r_{n} \rightarrow \infty$, then the MCMC procedure is said to have local weak consistency of order $r_{n}$. 
If an MCMC procedure has local consistency, then it has local weak consistency of order $r_{n} \equiv 1$. This order $\left\{r_{n}\right\}$ corresponds to the sufficient number of iterations to reach convergence, so a smaller $r_{n}$ is better.

The drawback of this property is that it is not easy to calculate in practice. In this paper, we consider another order for local degenerate MCMC procedures. According to Proposition 2.1 (i), the value $c_{n}\left(\theta_{1}-\theta_{0}\right)$ tends in probability to 0 . The order of degeneracy considers the rate of this convergence.

Definition 2.1.3 (Order of degeneracy). For positive sequence $d_{n}$, if

$$
c_{n} d_{n}\left|\theta_{1}-\theta_{0}\right|=O_{\mathbb{P}_{n}}(1),
$$

then $\left\{d_{n}\right\}$ is called the order of degeneracy of the MCMC procedure.

The order $d_{n}$ may not be optimal in general, that is, there may exist $\left\{c_{n}^{\prime}, d_{n}^{\prime}\right\}$ that satisfies $(2.2),(2.5)$, and $d_{n}^{\prime} / d_{n} \rightarrow \infty$. We do not treat the optimality issue in this paper to avoid tedious work for the proof.

Order of degeneracy is closely related to order of weak consistency. For a simple mixture model and a simple probit model, $d_{n}=r_{n}^{1 / 2}$ (see Kamatani (2013a, 2013b)), and this relation is probably always true in general under certain regularity conditions.

\subsection{Application to the DA procedure}

Now we focus on the DA procedure. Let $P(d x, d y \mid \theta)$ be a parametric family such that $P(d x \mid \theta)=\int_{y} P(d x d y \mid \theta)$. We call $P(d x \mid \theta)$ the observed-data model. Assume $x_{n} \sim P\left(d x_{n}\right)$ as in (2.1). The output $\theta_{\infty} \mid x_{n}$ is generated by the following iteration:

$$
y^{i}\left|\theta, x^{i} \sim P\left(d y^{i} \mid \theta, x^{i}\right)(i=1, \ldots, n), \quad \theta\right| x_{n}, y_{n} \sim P\left(d \theta \mid x_{n}, y_{n}\right) .
$$

Note that $x_{n}$ is fixed throughout the iteration, but that $\theta$ and $y_{n}$ are updated sequentially. For the related conditional distributions, we have the following property:

$$
P\left(d y_{n} \mid \theta, x_{n}\right) P\left(d \theta \mid x_{n}\right)=P\left(d \theta \mid x_{n}, y_{n}\right) P\left(d y_{n} \mid x_{n}\right) .
$$

First we note the following.

THEOREM 2.2.1 (Local consistency for DA). Under the regularity conditions in Appendix A, the DA procedure has local consistency.

See Kamatani (2014) for the proof. We state an equivalent condition for the orders of consistency and degeneracy for the DA procedure.

LEMma 2.1 (Sufficient condition for DA procedure). The order of consistency is $c_{n}$ if and only if there exists a $\mathbb{R}^{d}$-valued random variable $u_{n}\left(x_{n}\right)$ such that

$$
c_{n}\left|\theta_{0}-u_{n}\right|=O_{\mathbb{P}_{n}}(1)
$$


Moreover, if the MCMC procedure is a DA procedure and if the order of consistency is $c_{n}$, then $d_{n}$ is the order of degeneracy if and only if there exists a $\mathbb{R}^{d}$-valued random variable $v_{n}\left(x_{n}, y_{n}\right)$ such that

$$
c_{n} d_{n}\left|\theta_{0}-v_{n}\right|=O_{\mathbb{P}_{n}}(1) .
$$

Proof. The first claim directly follows from Lemma B.1 by applying $\mu_{n}=$ $P\left(d \theta \mid x_{n}\right)$ and $c_{n}=u_{n}\left(x_{n}\right)$.

For the latter claim, by (2.7), the conditional laws $\theta_{1}-v_{n} \mid x_{n}, y_{n}$ and $\theta_{0}-v_{n} \mid x_{n}, y_{n}$ are identical and independent, since

$$
\begin{aligned}
& P\left(d \theta_{1} \mid x_{n}, y_{n}\right) P\left(d y_{n} \mid \theta_{0}, x_{n}\right) P\left(d \theta_{0} \mid x_{n}\right) \\
& \quad=P\left(d \theta_{1} \mid x_{n}, y_{n}\right) P\left(d \theta_{0} \mid x_{n}, y_{n}\right) P\left(d y_{n} \mid x_{n}\right) .
\end{aligned}
$$

Therefore, as above, we can still apply Lemma B.1 for $\mu_{n}=P\left(d \theta \mid x_{n}, y_{n}\right)$ and $c_{n}=v_{n}\left(x_{n}, y_{n}\right)$, and hence the claim follows.

By the above fact, we may refer to $c_{n}$ as the order of consistency of the observed-data model, and $c_{n} d_{n}$ as that of the augmented-data model.

Suppose that $x^{i} \mid \theta \sim P\left(d x^{i} \mid \theta\right)(i=1, \ldots, n)$ and $\theta^{*} \mid x_{n} \sim P\left(d \theta^{*} \mid x_{n}\right)$ but that $\theta$ is fixed. Suppose also that

$$
\sqrt{n}\left(\theta^{*}-u_{n}\right) \mid \theta \rightarrow N\left(0, I(\theta)^{-1}\right)
$$

for any $\theta$, where $I(\theta)$ is a positive definite matrix, and $u_{n}\left(x_{n}\right)$ is a $\mathbb{R}^{d}$-valued random variable. This is a conclusion of the Bernstein-von Mises theorem. If the above holds, (2.8) is satisfied by integrating out $\theta$. Hence if the Bernstein-von Mises theorem holds, then (2.8) holds by Lemma B.1.

We also call attention to a (subjective) Bayesian trick. As above, tightness of $\sqrt{n}\left(\theta^{*}-u_{n}\right) \mid \theta$ for each $\theta$ is a sufficient condition for (2.8). However, thanks to the subjective Bayes setting (2.1), tightness of $\sqrt{n}\left(\theta^{*}-u_{n}\right) \mid \theta^{*}$ for each $\theta^{*}$ is sufficient. The latter requires no posterior calculation. To see this sufficiency, observe

$$
\begin{aligned}
\int f\left(\theta^{*}, x_{n}\right) P\left(d x_{n} \mid \theta^{*}\right) P\left(d \theta^{*}\right) & =\int f\left(\theta^{*}, x_{n}\right) P\left(d \theta^{*} \mid x_{n}\right) P\left(d x_{n}\right) \\
& =\int f\left(\theta^{*}, x_{n}\right) P\left(d \theta^{*} \mid x_{n}\right) P\left(d x_{n} \mid \theta\right) P(d \theta)
\end{aligned}
$$

and apply $f\left(\theta, x_{n}\right)=\min \left\{\sqrt{n}\left|\theta-u_{n}\right|>M_{n}, 1\right\}$ for $M_{n} \rightarrow \infty$. This argument is summarized as follows.

Lemma 2.2 (Sufficient condition for DA procedure by Bayesian trick).

(i) If $c_{n}\left(\theta-u_{n}\right) \mid \theta$ is tight for each $\theta$ for some $u_{n}\left(x_{n}\right)$, then the order of consistency of the observed-data model is $c_{n}$.

(ii) Suppose that the $M C M C$ procedure is the DA procedure. If $c_{n} d_{n}\left(\theta-v_{n}\right) \mid \theta$ is tight for each $\theta$ for some $v_{n}\left(x_{n}, y_{n}\right)$, then the order of consistency of the augmented-data model is $c_{n} d_{n}$. 


\subsection{First example}

We first consider the example in the Introduction to show that the calculation of order of degeneracy is easy. Assume (1.2) with prior $\theta \sim N(0,1)$. The augmented-data model (1.3) defines the following DA procedure:

$$
\left\{\begin{array}{l}
\text { simulate } y^{i} \mid \theta, x^{i} \sim\left\{\begin{array}{ll}
N(0,1,-\infty, \theta) & \text { if } x^{i}=1 \\
N(0,1, \theta, \infty) & \text { if } x^{i}=0
\end{array} \quad(i=1, \ldots, n),\right. \\
\text { simulate } \theta \mid x_{n}, y_{n} \sim N\left(0,1, \max _{i: x^{i}=1} y^{i}, \min _{i: x^{i}=0} y^{i}\right),
\end{array}\right.
$$

where $N(0,1, a, b)$ is the standard normal distribution truncated to $[a, b]$. By Lemma $2.2(\mathrm{i})$, it is easy to prove that $c_{n}=\sqrt{n}$. To see this, consider reparametrization $\tilde{\theta}=\Phi(\theta) \in[0,1]$; prove $\sqrt{n}\left(\tilde{\theta}-\tilde{u}_{n}\right)=O_{\mathbb{P}_{n}}(1)$ for $\tilde{u}_{n}=n_{1} / n$ and then make a projection by $\Phi^{-1}$, where $n_{1}$ is the number of observation of $x^{i}=1$. For $v_{n}=\max _{i ; x^{i}=1} y^{i}$, we prove $d_{n}=\sqrt{n}$ by using Lemma 2.2 (ii). To see this, take $M=-\log \epsilon / \phi(\theta)$ for $\epsilon>0$; then by $n_{1} / n \rightarrow \Phi(\theta)$, we have

$$
\begin{aligned}
\mathbb{P}_{n}\left(n\left(v_{n}-\theta\right)<-M \mid \theta\right) & =\mathbb{P}_{n}\left(\prod_{i ; x^{i}=1}^{n} \mathbb{P}_{n}\left(y^{i}<\theta-\frac{M}{n} \mid \theta, x^{i}\right) \mid \theta\right) \\
& =\mathbb{E}_{n}\left[\left(\frac{\Phi\left(\theta-\frac{M}{n}\right)}{\Phi(\theta)}\right)^{n_{1}}\right] \theta
\end{aligned}
$$

and this tends to $e^{-\phi(\theta) M}=\epsilon$.

On the other hand for the model (1.4), $\theta \mid x_{n}, y_{n}$ is $N\left(-\sum_{i=1}^{n} y_{i} /(n+\right.$ $1), 1 /(n+1))$. Hence by $(2.10), \theta_{1} \mid x_{n}, y_{n}$ and $\theta_{0} \mid x_{n}, y_{n}$ are independent and

$$
n^{1 / 2}\left(\theta_{1}-\theta_{0}\right) \mid x_{n}, y_{n} \sim N\left(0, \frac{n}{n+1}\right) \rightarrow N(0,1) .
$$

Therefore, this DA procedure cannot have local degeneracy by Proposition 2.1.

\subsection{Estimation from the data}

Order of degeneracy is determined by the model. Estimation of it from the simulated data is difficult, but is still possible. For simplicity, assume $M$ to be an even number. For $\theta_{0}, \theta_{1}, \ldots, \theta_{M-1}$,

$$
\hat{d}=\frac{\gamma\left(\left\{\left|\theta_{m}-\theta_{m+M / 2}\right| ; m=0, \ldots, M / 2-1\right\}\right)}{\gamma\left(\left\{\left|\theta_{m}-\theta_{m+1}\right| ; m=0, \ldots, M-2\right\}\right)}
$$

where $\gamma\left(\left\{s_{i}\right\}\right)$ is the median of a sequence $\left\{s_{i}\right\}$. This has a close connection to the empirical autocorrelation and the effective sample size. If the Markov chain $\theta_{\infty} \mid x_{n}$ is ergodic, this tends to the ratio of the medians of $P\left(\left|\theta_{0}-\theta_{1}\right| \in \cdot \mid x_{n}\right)$ and $P\left(\left|\theta-\theta_{*}\right| \in \cdot \mid x_{n}\right)$ where $\theta$ and $\theta_{*}$ are as in Definition 2.1.1. Therefore if 

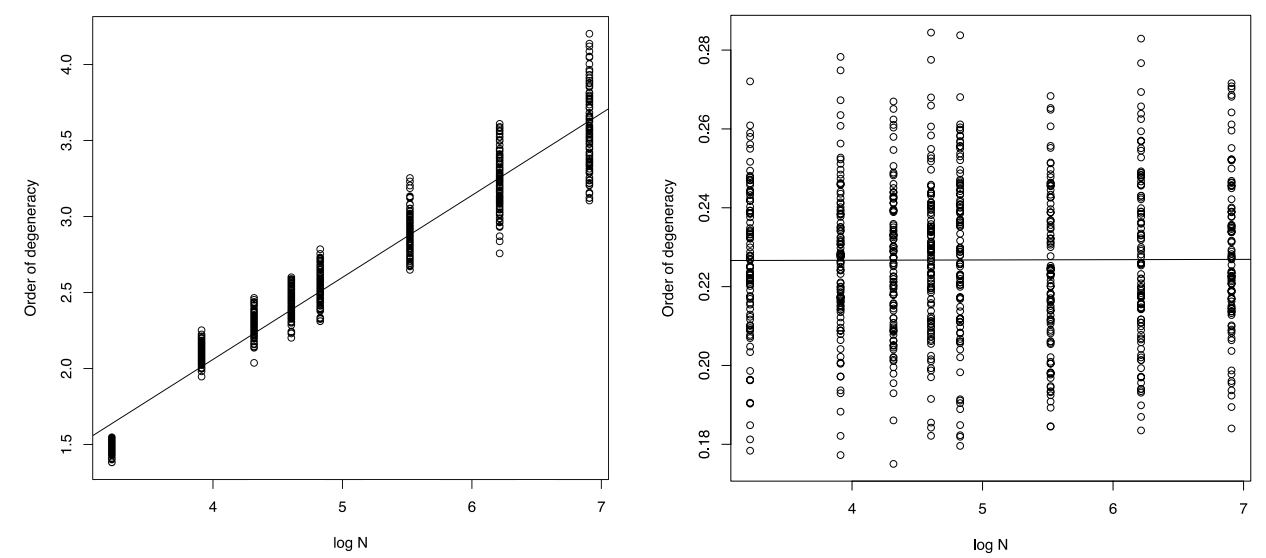

Figure 2. Plots of estimates of order of degeneracy in log scale. DA procedures are those for the model (1.2) with the augmented-data models (1.3) (left) and (1.4) (right).

the two medians are of orders $c_{n}$ and $c_{n} d_{n}$, respectively, then the estimator is of order $d_{n}$ for a sufficiently large $M$. For example, for the two DA procedures in the Introduction we have the following results. We calculate 8 sample sizes $\{25,50,75,100,125,250,500,1000\}$ for $M=10^{4}$ iterations with 100 trials for each. The results are shown in Figure 2, where the $x$ coordinate is the log sample size and the $y$ coordinate is $\log \hat{d}$. The estimated regression lines (solid lines) are $y=-0.099 \cdots+x 0.5398 \cdots$ (left) and $y=0.2264 \cdots+x 0.00007 \cdots$ (right). Since the orders of degeneracy for the augmented-data models (1.3) and (1.4) are $\sqrt{n}$ and 1 , the slopes should be 0.5 and 0 in the limit, respectively. Hence, this result is reasonable.

Note that for the Metropolis-Hastings procedure, another measure, such as the $\alpha$-percentile $(\alpha<50)$, instead of the median may provide a better estimate of the order of degeneracy.

The estimate $\hat{d}$ is considered to be a kind of convergence diagnostics for MCMC. A useful review of such diagnostics can be seen in Cowles and Carlin (1996). Our main interest here is the theoretical properties of the order of degeneracy and not its estimation or convergence diagnostics, and so we do not treat it further in this paper.

\section{Application}

\subsection{One of the explanatory variables is dominant}

For a regression model for categorical data, sometimes the explanatory variable has too strong an effect on the observation, which results in a large variance in estimation. On the other hand, for the augmented-data model, the converse effect may appear, that is, estimation becomes relatively accurate. These two effects slow down the convergence of the DA procedure that is suggested by Lemma 2.2. We refer to this as the dominance of the explanatory variables. Let $\epsilon>0$. 
Consider the model

$$
P(y=1 \mid \theta, x)=1-P(y=-1 \mid \theta, x)=\Phi(\theta x)
$$

with $x \sim N\left(0, \epsilon^{-2}\right)$ and the prior distribution $\theta \sim N(0,1)$. We let $\epsilon \rightarrow 0$, which makes the observation less informative since the Fisher information matrix becomes smaller. We will see that the order of consistency is not the usual rate $\sqrt{n}$ but $\sqrt{\epsilon n}$. The augmented data model is

$$
y=1_{\{z \leq 0\}}-1_{\{z>0\}}, \quad z \mid \theta, x \sim N(-\theta x, 1), \quad x \sim N\left(0, \epsilon^{-2}\right) .
$$

Surprisingly, for this augmented-data model, it becomes more informative as $\epsilon \rightarrow 0$. More precisely, the order of consistency for the augmented-data model is not the usual rate $\sqrt{n}$ but $\epsilon^{-1} \sqrt{n}$. The corresponding DA procedure is the following iteration:

$$
\begin{aligned}
& \text { simulate } z^{i} \mid \theta, x^{i}, y^{i} \sim\left\{\begin{array}{l}
N\left(-\theta x^{i}, 1,0, \infty\right) \quad \text { if } y^{i}=-1 \\
N\left(-\theta x^{i}, 1,-\infty, 0\right) \quad \text { if } y^{i}=1
\end{array} \text { for } i=1, \ldots, n,\right. \\
& \text { simulate } \theta \quad \mid \quad x_{n}, y_{n}, z_{n} \sim N\left(-\sum_{i=1}^{n} z^{i} x^{i} /\left(1+\sum_{i=1}^{n}\left(x^{i}\right)^{2}\right), 1 /(1+\right. \\
& \left.\left.\sum_{i=1}^{n}\left(x^{i}\right)^{2}\right)\right) .
\end{aligned}
$$

To make the analysis easier, we simplify the model. Let $\operatorname{sign}(x)=x /|x|$. Since $1(\{y=\operatorname{sign}(x)\})$ and $|x|$ are sufficient statistics for the estimation of $\theta,(3.1)$ can be written as

$$
P(y=\operatorname{sign}(x) \mid \theta, x)=1-P(y \neq \operatorname{sign}(x) \mid \theta, x)=\Phi(\theta|x|),
$$

and hence we may assume instead the model (3.1) with $x \sim N\left(0, \epsilon^{-2}, 0, \infty\right)$ and prior $N(0,1)$. Let $\phi^{+}(x)=2 \phi(x) 1_{\{x>0\}}$, and let $c(x)=1 / 2+\arctan (x) / \pi$, which is the cumulative distribution function of the Cauchy distribution. Then

$$
\begin{aligned}
P(y=1 \mid \theta) & =\int \Phi(\theta x / \epsilon) \phi^{+}(x) d x \\
& =\int 1(\delta \leq \theta x / \epsilon) \phi^{+}(x) \phi(\delta) d x d \delta=c(\theta / \epsilon)
\end{aligned}
$$

since $\delta /|x|$ follows the Cauchy distribution if $\delta, x \sim N(0,1)$. We show that in the current setting, the DA procedure has poor performance.

Proposition 3.2. Let $\epsilon \rightarrow 0$ but $\epsilon n \rightarrow \infty$. The DA procedure has local degeneracy, and the order of degeneracy is $\epsilon^{-3 / 2}$.

Proof. Let $n_{1}$ denote the number of outcomes of $y^{i}=1$ and set $c_{n}=\sqrt{\epsilon n}$ and $u_{n}=\epsilon c^{-1}\left(n_{1} / n\right)$. Let us denote $X_{n}=O_{\mathbb{P}_{n} \mid \theta}(1)$ if $X_{n} \mid \theta$ is tight. By Lemma 2.2 (i), the order of consistency is $c_{n}$ if

$$
c_{n}\left|\theta-u_{n}\right|=O_{\mathbb{P}_{n} \mid \theta}(1)
$$


for each $\theta$. Since $c(-x)=O\left(x^{-1}\right)(x \rightarrow \infty)$ and $\epsilon n \rightarrow \infty$, by checking the Lindeberg condition, the central limit theorem holds for $n_{1}$ (see Example 2.4.8 of Lehmann (1998)):

$$
\xi_{n}:=\frac{n_{1}-n c(\theta / \epsilon)}{\sqrt{n c(\theta / \epsilon) c(-\theta / \epsilon)}} \mid \theta \rightarrow N(0,1) .
$$

Therefore, $n_{1} / n=c(\theta / \epsilon)+O_{\mathbb{P}_{n} \mid \theta}\left(\epsilon^{1 / 2} / \sqrt{n}\right)$. Thus, by $\left(c^{-1}\right)^{\prime}(c(x))=\pi\left(1+x^{2}\right)$,

$$
u_{n}=\epsilon c^{-1}\left(c(\theta / \epsilon)+O_{\mathbb{P}_{n} \mid \theta}\left(\epsilon^{1 / 2} / \sqrt{n}\right)\right)=\theta+O_{\mathbb{P}_{n} \mid \theta}\left(1 / c_{n}\right),
$$

which proves that the order of consistency is $c_{n}=\sqrt{\epsilon n}$.

Next we check the order of degeneracy. Generate $\theta \mid x_{n}, y_{n}, z_{n}$ and $\theta_{*} \mid$ $x_{n}, y_{n}, z_{n}$ independently. Then by construction,

$$
\theta-\theta_{*} \mid x_{n}, y_{n}, z_{n} \sim N\left(0, \frac{2}{1+\sum_{i=1}^{n}\left(x^{i}\right)^{2}}\right) .
$$

Therefore, $\theta-\theta_{*}=O_{\mathbb{P}_{n}}\left(\epsilon^{2} / n\right)$ since $\left(\sum_{i=1}^{n}\left(x^{i}\right)^{2}\right)^{-1}=O_{\mathbb{P}_{n} \mid \theta}\left(\epsilon^{2} / n\right)$. Hence by Lemma B.1, there exists $v_{n}\left(x_{n}, y_{n}, z_{n}\right)$ such that $\epsilon^{-1} \sqrt{n}\left(\theta_{0}-v_{n}\right)=O_{\mathbb{P}_{n}}(1)$ and we have $d_{n}=\left(\epsilon^{-1} \sqrt{n}\right) / \sqrt{\epsilon n}=\epsilon^{-3 / 2}$.

As in this example, the DA procedure sometimes suffers from slow convergence. Surprisingly, in some cases, this can be drastically improved by adding a single working parameter. Strategies that do this include the parameterexpanded data augmentation (PX-DA) procedure proposed by Liu and Wu (1999) and the marginal augmentation (MA) procedure proposed by Meng and van Dyk (1999). These two procedures have essentially the same asymptotic property, so we only consider the PX-DA procedure. In the current case, the PX-DA procedure is the following:

simulate $\theta$ as the DA procedure,

simulate $\gamma^{2} \mid \theta, x_{n}, y_{n}, z_{n}$ from $\operatorname{Gamma}\left(2^{-1}(n+1), 2^{-1}\left(\sum_{i=1}^{n}\left(z^{i}+\theta x^{i}\right)^{2}+\right.\right.$ $\left.\theta^{2}\right)$ ),

output $\gamma \theta$, where $\operatorname{Gamma}(\nu, \alpha)$ is the Gamma distribution with shape parameter $\nu$ and rate parameter $\alpha$. As the next proposition shows, the order of degeneracy becomes much better with the PX-DA procedure.

Proposition 3.3. Under the same setting as Proposition 3.2, the PX-DA procedure has order of degeneracy $\epsilon^{-1 / 2}$.

Proof. We use the same notation as in the proof of Proposition 3.2. Since $z^{i}+\theta x^{i} \mid \theta, x_{n} \sim N(0,1)$, we have $\gamma^{2}=1+O_{\mathbb{P}_{n} \mid \theta}\left(n^{-1 / 2}\right)$. Thus,

$$
\sqrt{n}\left|\gamma \theta_{1}-\theta_{0}\right| \leq \sqrt{n}\left(\gamma\left|\theta_{1}-\theta_{0}\right|+|\gamma-1|\left|\theta_{0}\right|\right)=O_{\mathbb{P}_{n} \mid \theta_{0}}(1) .
$$

Hence the PX-DA procedure still has local degeneracy, but has $d_{n}=\sqrt{n} / \sqrt{\epsilon n}=$ $\epsilon^{-1 / 2}$. 
Using a slightly different model, Omori (2007) considered a partially observed linear regression model where two of three parameters in $\theta$ were large, and he reported that the DA procedure performed badly in that setting, but the marginal augmentation strategy worked fairly well. Also, in Liu and Wu (1999, p. 1273), a similar property was reported. Imai and van Dyk (2005) reported the poor performance of the DA procedure used in McCulloch et al. (2000) and that the convergence was improved by the PX-DA procedure. These are due to the dominance of the explanatory variables.

This model was well studied in terms of operator norm and efficient ordering by Roy and Hobert (2007). Our results here explain the mechanism of superiority of the PX-DA procedure. Moreover, we obtain different orders of degeneracy. Note that when $\epsilon$ is constant, both the DA and the PX-DA procedures have local consistency, and hence the order of degeneracy is 1 .

\subsection{Fragility of identification}

Nonidentifiability of the parameters results in difficulties for statistical inference. Models with missing data, including mixture models, sometimes have similar difficulties even when the parameters are identifiable. In Keane (1992), it was reported that parameter inference for the multinomial probit model is difficult due to the fragility of identifying the parameters. The fragility occurs when the contributions (amount of information) to the model of some parameters are small. The DA procedure also suffers from the same problem. To analyze the effect on the DA procedure, consider the two-component mixture model

$$
x^{i} \mid \theta \sim \theta N(\epsilon, 1)+(1-\theta) N(0,1), \quad i=1, \ldots, n, \quad \theta \sim \operatorname{Beta}\left(\alpha_{0}, \alpha_{1}\right),
$$

where $\alpha_{0}, \alpha_{1}>0$ are known and $\epsilon \rightarrow 0$. In the limit $\epsilon=0$, the model loses identifiability, but if $\epsilon>0$, it still has identifiability. A natural DA procedure is

$$
\left\{\begin{array}{l}
y^{i} \mid \theta, x^{i} \sim \operatorname{Bernoulli}\left(\frac{\theta \phi\left(x^{i}-\epsilon\right)}{\theta \phi\left(x^{i}-\epsilon\right)+(1-\theta) \phi\left(x^{i}\right)}\right) \\
\theta \mid x_{n}, y_{n} \sim \operatorname{Beta}\left(\alpha_{0}+n_{0}, \alpha_{1}+n_{1}\right),
\end{array} \quad(i=1, \ldots, n),\right.
$$

where $\phi$ is the density function of $N(0,1)$ and $n_{j}=\sum_{i=1}^{n} 1_{\left\{y^{i}=j\right\}}$ for $j=0,1$.

Proposition 3.4. Assume $\epsilon \sqrt{n} \rightarrow \infty$ but $\epsilon \rightarrow 0$. Then the DA procedure has order of degeneracy $\epsilon^{-1}$.

Proof. Let $u_{n}=\sum_{i=1}^{n} x^{i} / \epsilon n$. Then by checking the Lindeberg condition, $\epsilon \sqrt{n}\left(\theta-u_{n}\right) \mid \theta \rightarrow N(0,1)$, and hence the order of consistency is $c_{n}=\epsilon \sqrt{n}$ by Lemma 2.2 (i). The law $n_{1} \mid \theta$ is the binomial distribution with parameter $\theta$ and number of trials $n$. Hence for $v_{n}=n_{1} / n$, we have a tightness of $\sqrt{n}\left(\theta-v_{n}\right) \mid \theta$, and the order of degeneracy is $\sqrt{n} / \epsilon \sqrt{n}=\epsilon^{-1}$ by Lemma 2.2 (ii).

Sometimes it is possible to find efficient MCMC procedures, as we did in Kamatani (2013a). Another way is to eliminate parameters that are not important for the description of the model. 


\subsection{Overly large Fisher information matrix}

Sometimes the order of degeneracy differs between parameters. For such cases, we slightly extend the definition of order of degeneracy. For $\varphi: \Theta \rightarrow \mathbb{R}^{k}$ for some $k \in \mathbb{N}$, if $c_{n}\left|\varphi(\theta)-\varphi\left(\theta_{*}\right)\right|=O_{\mathbb{P}_{n}}(1)$ and $c_{n} d_{n}\left|\varphi\left(\theta_{0}\right)-\varphi\left(\theta_{1}\right)\right|=O_{\mathbb{P}_{n}}(1)$, we call $c_{n}$ and $d_{n}$ the order of $\varphi$-consistency and the order of $\varphi$-degeneracy, respectively.

In some cases, the augmented-data model has much larger information than that of the observed-data model. It is well known that this causes a problem for the DA procedure (see, e.g., Dempster et al. (1977) for an early mention of this problem, although this reference is for the case of the EM algorithm). One such example is the cumulative probit model

$$
P(y \leq j \mid \theta, x)=\Phi\left(\alpha_{j}+\beta^{T} x\right) \quad(j=1,2, \ldots, c-1), \quad x \sim G(d x)
$$

where $\theta=(\alpha, \beta)=\left(\alpha_{2}, \ldots, \alpha_{c-1}, \beta\right) \in \mathbb{R}^{c-2} \times \mathbb{R}^{d}$ and $\beta^{T}$ is the transpose of a vector $\beta \in \mathbb{R}^{d}$. Assume that $G(d x)$ has compact support. The parameter $\alpha$ satisfies $0=$ : $\alpha_{1}<\alpha_{2}<\cdots<\alpha_{c-1}<\alpha_{c}:=\infty$. The following lemma follows by the usual regularity conditions. See the Appendix of Kamatani (2011) for details.

LEMMA 3.3. If the expectation of $\left(1, x^{T}\right)\left(\begin{array}{l}1 \\ x\end{array}\right)$ for $x \sim G(d x)$ is a non-degenerate matrix, then $\sqrt{n}\left(\alpha-u_{n}\right)=O_{\mathbb{P}_{n}}(1)$ for some $u_{n}\left(x_{n}, y_{n}\right)$.

By the above, the observed-data model has the usual order of consistency $\sqrt{n}$. However, its augmented-data model

$$
z \mid \theta, x, y \sim N\left(-\beta^{T} x, 1\right) \quad \text { and } \quad y=j \quad \text { if } z \in\left(\alpha_{j-1}, \alpha_{j}\right]
$$

has a different order of consistency since it has parameter-dependent support. A comparison of the DA and the PX-DA procedures was made in Liu and Sabatti (2000) and their local properties were studied in Kamatani (2011). The DA procedure iterates the following:

simulate $z^{i} \mid \theta, x^{i}, y^{i} \sim N\left(-\beta^{T} x^{i}, 1, \alpha_{y^{i}-1}, \alpha_{y^{i}}\right)$ for $i=1, \ldots, n$,

simulate $\alpha_{j} \mid y_{n}, z_{n}$ from $N\left(0,1, \max _{i ; y^{i}=j} z^{i}, \min _{i ; y^{i}=j+1} z^{i}\right)$ for $j=2, \ldots$, $c-1$,

simulate $\beta \mid x_{n}, z_{n}$ from $N\left(-\left(1+\sum_{i=1}^{n} x^{i}\left(x^{i}\right)^{T}\right)^{-1} \sum_{i=1}^{n} x^{i} z^{i} /(n+1)\right.$, $1 /(n+1))$.

Proposition 3.5. Take $\varphi(\theta)=\alpha$. Then the DA procedure has order of $\varphi$-degeneracy $\sqrt{n}$.

Proof. Let $c_{n}=\sqrt{n}$. By Lemma 2.2 (i) together with Lemma 3.3, the order of consistency is $\sqrt{n}$. By Lemma 2.2 (ii), to prove $d_{n}=\sqrt{n}$, it suffices to show the tightness of $n\left(\alpha_{j}-v_{n j}\right) \mid \theta$ for $v_{n j}=\max _{i ; y^{i}=j} z^{i}$ for each $j=2, \ldots, c-1$. By assumption, $\operatorname{supp} G$, the support of $G$, is bounded and hence there exists 
$c=c(\theta)>0$ such that $\phi\left(\beta^{T} x+\alpha_{j}+\epsilon\right) \in(c, 1)(x \in \operatorname{supp} G,|\epsilon|<1)$ for each parameter $\theta$. Take $M>0$. We may assume $|M / n|<1$. Then

$$
\begin{aligned}
\mathbb{P}_{n}\left(n\left(v_{n j}-\alpha_{j}\right)<-M \mid \theta\right) & \\
= & \mathbb{P}_{n}\left(\prod_{i ; y^{i}=j} \mathbb{P}_{n}\left(z^{i}<\alpha_{j}-\frac{M}{n} \mid \theta, x^{i}, y^{i}\right) \mid \theta\right) \\
= & \mathbb{E}_{n}\left[\prod_{i ; y^{i}=j} \frac{\Phi\left(\beta^{T} x^{i}+\alpha_{j}-\frac{M}{n}\right)-\Phi\left(\beta^{T} x^{i}+\alpha_{j-1}\right) \mid}{\Phi\left(\beta^{T} x^{i}+\alpha_{j}\right)-\Phi\left(\beta^{T} x^{i}+\alpha_{j-1}\right)} \mid \theta\right] \\
\leq & \mathbb{E}_{n}\left[\exp \left(-\sum_{i ; y^{i}=j} \frac{\Phi\left(\beta^{T} x^{i}+\alpha_{j}\right)-\Phi\left(\beta^{T} x^{i}+\alpha_{j}-\frac{M}{n}\right)}{\Phi\left(\beta^{T} x^{i}+\alpha_{j}\right)-\Phi\left(\beta^{T} x^{i}+\alpha_{j-1}\right)}\right) \mid \theta\right] \\
\leq & \mathbb{E}_{n}\left[\exp \left(-\frac{c M}{\alpha_{j}-\alpha_{j-1}} \frac{n_{j}}{n}\right) \mid \theta\right]
\end{aligned}
$$

where $n_{j}$ is number of outcomes of $y^{i}=j$. Since $n_{j} / n \mid \theta$ is tight, $n\left(v_{n j}-\alpha_{j}\right) \mid \theta$ is also tight. Hence the claim follows.

In the present case, the PX-DA procedure adds the following step after each iteration of the DA procedure:

$$
\left\{\begin{aligned}
\text { simulate } \gamma^{2} \sim \operatorname{Gamma} & \frac{n+d+c}{2}-1, \\
& \left.\frac{1}{2}\left(\sum_{i=1}^{n}\left(z^{i}+\beta^{T} x^{i}\right)^{2}+|\beta|^{2}+\sum_{j=2}^{c-1} \alpha_{j}^{2}\right)\right), \\
\text { set } \quad \theta \leftarrow \gamma \theta &
\end{aligned}\right.
$$

The following proposition shows that even if the PX-DA procedure seems to be fine, it can have local degeneracy. The key fact is that projection such as $\varphi(\theta)=\alpha_{3} / \alpha_{2}$ cancels out the benefit of the PX-DA procedure, and hence $\varphi(\theta)$ has almost the same behavior as that of the DA procedure. The proof is a corollary of the above proposition and we omit it (see Kamatani (2011) for details).

Proposition 3.6. Take $\varphi(\theta)=\alpha_{3} / \alpha_{2}$. Then the $P X-D A$ procedure has order of degeneracy $\sqrt{n}$ when $c>3$.

For this model, the PX-DA procedure does not work well. However by introducing Metropolis-Hastings steps, it is possible to construct an MCMC with local consistency even for this model. Details will be presented elsewhere. The important point is that this degeneracy is not directly visible since the PX-DA 

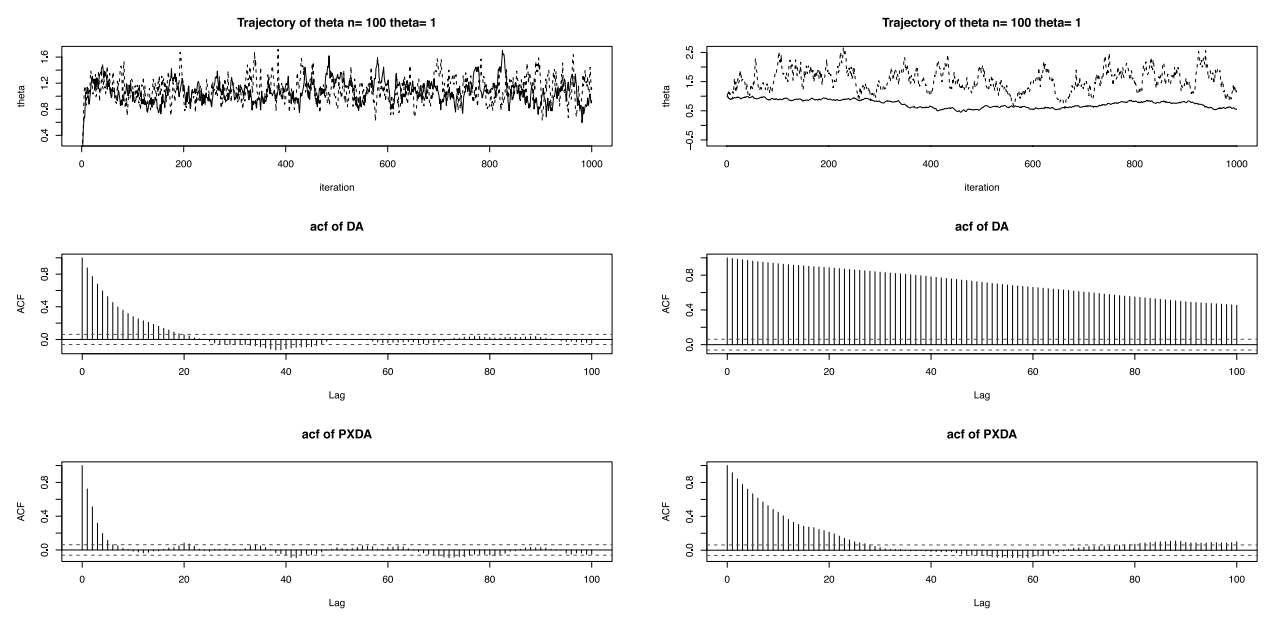

Figure 3. Model (3.1) with $x \sim N\left(0, \epsilon^{-2}\right)$ and sample size $n=100$. The left figures are for $\epsilon=0.5$, and those on the right are for $\epsilon=0.1$.
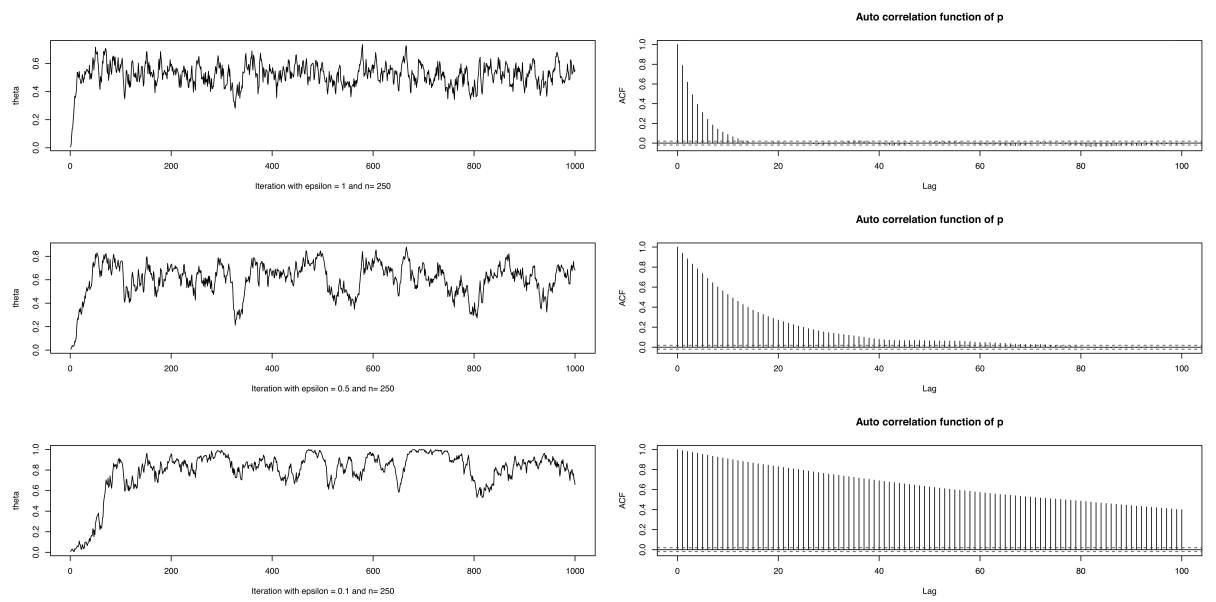

Figure 4. Model (3.7) for $\epsilon=1$ (top), 0, 5 (middle), and 0.01 (bottom) with sample size $n=250$. The left figures are trajectories, and the right figures are empirical autocorrelations.

does have local degeneracy under $\varphi(\theta)=\theta$. The degeneracy appears when we set a suitable projection ( $\operatorname{such}$ as $\varphi(\theta)=\alpha_{3} / \alpha_{2}$ as above). It is straightforward to find such a projection with the knowledge of the local property, the order of degeneracy.

\section{Simulation}

First we consider the probit model (3.1) with small $\epsilon$ considered in Subsection 3.1. Let the true parameter be $\theta=1$, the sample size $n=100$, and $\epsilon=0.5$ (lefthand side of Figure 3) and $\epsilon=0.1$ (right). By Proposition 3.3, the PX-DA procedure improves the order from $\epsilon^{-3 / 2}$ (DA) to $\epsilon^{-1 / 2}$ (PX-DA). As theoretical 

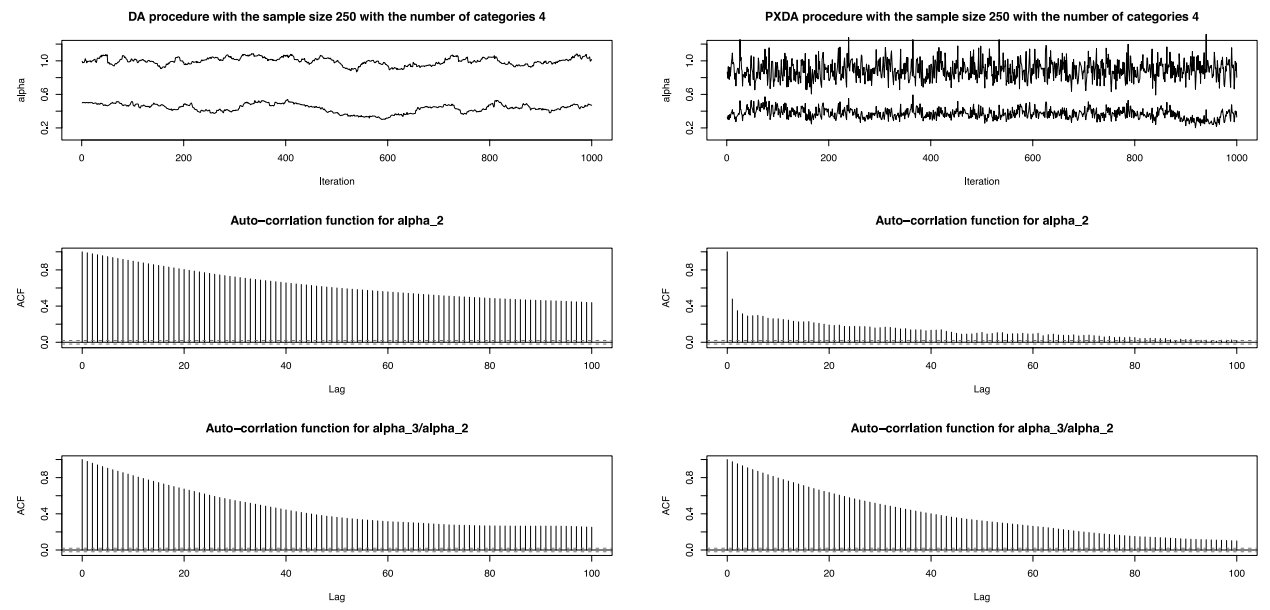

Figure 5. Cumulative probit model with sample size $n=250$ for the DA (left) and the PX-DA (right) procedures. Trajectory and empirical autocorrelation for $\alpha_{2}$ for the PX-DA procedure (the top right and middle right figures) seem to be much better than those of the DA procedure (the top left and the middle left figures). However, a projection $\alpha_{3} / \alpha_{2}$ cancels out the benefit (bottom figures).

results suggest, the performance of the DA procedure is worse than that of the PX-DA procedure, and the difference becomes larger as $\epsilon$ becomes smaller. On the other hand, if $\epsilon$ is not so small, the difference is not so large.

Next we consider (3.7) with the fragility of identification as described in Subsection 3.2. We consider the three cases $\epsilon=1,0.5$, and 0.01 with sample size $n=250$. As suggested by Proposition 3.4, the DA procedure has poor performance when $\epsilon$ is small. Therefore, the results for $\epsilon=0.01$ should be worse than those of $\epsilon=1$. The effect of $\epsilon$ on the performance seems milder than in the previous example, as suggested by the theoretical results. To be more speficic, the order of degeneracy of the DA procedure is $\epsilon^{-3 / 2}$ for the previous case, and $\epsilon^{-1}$ for the current case (note that conditions $\epsilon n \rightarrow \infty$ for the former and $\epsilon \sqrt{n} \rightarrow \infty$ for the latter should be satisfied).

Finally, we consider the cumulative probit model in Subsection 3.3. The number of categories is $c=4$, and so the DA procedure and the PX-DA procedure with $\varphi(\theta)=\alpha_{3} / \alpha_{2}$ have local degeneracy with order $\sqrt{n}$. At a first glance, the PX-DA procedure may seem to be the much better of the two. However, as suggested by the theoretical results in Subsection 3.3, the PX-DA procedure still has poor mixing properties, as we can see in the bottom figure of Figure 5 . It seems that simple diagnostics such as autocorrelation plots of $\alpha$ may fail to find poor behavior. However, considering local properties by using the order of degeneracy makes it straightforward to find.

\section{Concluding remarks}

The present paper studies the order of degeneracy using the local properties of MCMC procedures. As an application, we considered three models. As shown 
in Section 4, the order of degeneracy explains a part of the real behavior of MCMC procedures. This property is relatively easy to use, and it can measure the inefficiency of MCMC procedures. Once this inefficiency is identified, it is not difficult to eliminate or reduce it, as shown by Kamatani (2013a).

\section{Acknowledgements}

The author thanks the Associate Editor and an anonymous referee for constructive comments which were much help in improving this paper. This work is supported in part by Grant-in-Aid for Young Scientists (B) 24740062.

Appendix A: Regularity condition

Suppose that $\Theta=\mathbb{R}^{d}$ and that $P(d x \mid \theta)$ has probability distribution function $p(x \mid \theta)$. Write $f_{h}=o(h)$ in $L^{2}$ if $|h|^{-2} \int\left|f_{h}(x)\right|^{2} d x \rightarrow 0$ for $h \rightarrow 0$. If

$$
\sqrt{p(x \mid \theta+h)}-\sqrt{p(x \mid \theta)}-h^{T} \tilde{\eta}(x)=o(h) \quad \text { in } \quad L^{2}
$$

for any $h \rightarrow 0$ for a square integrable function $\tilde{\eta}(x)$, we refer to the parametric family as quadratic mean differentiable. This condition is quite general and easily checked by, for example, the criterion given in Hajek (1972). See Section 12 of Lehmann and Romano (2005) for further details.

We also assume a kind of separation condition. A sequence of tests $\psi_{n}\left(x_{n}\right) \in$ $[0,1]$ is said to be uniformly consistent for $\theta \in \mathbb{R}^{d}$ against $K \subset \mathbb{R}^{d}$ if

$$
\mathbb{E}\left(\psi_{n}\left(x_{n}\right) \mid \theta\right) \rightarrow 0, \quad \sup _{\vartheta \in K^{c}} \mathbb{E}\left(1-\psi_{n}\left(x_{n}\right) \mid \vartheta\right) \rightarrow 0 .
$$

If there exists a uniformly consistent test for any $\theta$ and some compact set $K$, then a parametric family is said to have a uniformly consistent test.

The following is a sufficient condition for local consistency of the DA procedure.

(i) $P(d x, d y \mid \theta)$ is quadratic mean differentiable and it has a density $p(x, y \mid \theta)$ such that $p(x, y \mid \theta)>0$.

(ii) The Fisher information matrix of $P(d x \mid \theta)$ is non-singular.

(iii) $P(d x \mid \theta)$ has a uniformly consistent test.

(iv) The prior distribution has a continuous, positive, and bounded density.

(v) $P(d x \mid \theta)$ has identifiability.

\section{Appendix B: Tightness condition}

We need the following theorem to show equivalent conditions for degeneracy. We will apply it for $\mu_{n}=P\left(d \theta \mid x_{n}\right)$ and hence it depends on $x_{n}$. In this case, $c_{n}$ depends on $x_{n}$, but still we can apply the following lemma.

Lemma B.1. Let $\left(\mu_{n}\right)_{n \in \mathbb{N}}$ be a sequence of probability measures on $\mathbb{R}^{k}$. Suppose that $X_{n}$ and $X_{n}^{\prime}$ are i.i.d. draws from $\mu_{n}$; then $\left|X_{n}-X_{n}^{\prime}\right|=O_{\mathbb{P}}(1)$ if and only if there exists $c_{n} \in \mathbb{R}^{k}$ such that $\left|X_{n}-c_{n}\right|=O_{\mathbb{P}}(1)$.

Proof. Sufficiency of $\left|X_{n}-c_{n}\right|=O_{\mathbb{P}}(1)$ is clear by the triangular inequality. We show necessity as follows. Without loss of generality, we can assume 
$k=1$. Let $x^{+}=\max \{x, 0\}$ and $x^{-}=\max \{-x, 0\}$. By definition, $x=x^{+}-x^{-}$, and if $x^{+}>0$, then $x^{-}=0$ and vice versa. Let $c_{n}$ be the median of $\mu_{n}$, that is,

$$
\mathbb{P}\left(Y_{n}^{-}=0\right) \geq 1 / 2 \quad \text { and } \quad \mathbb{P}\left(Y_{n}^{+}=0\right) \geq 1 / 2,
$$

where $Y_{n}=X_{n}-c_{n}$. Therefore, since $Y_{n}=Y_{n}^{+}-Y_{n}^{-}$, for $M>0$,

$$
\begin{aligned}
\mathbb{P}\left(X_{n}-X_{n}^{\prime}>M\right) & =\mathbb{P}\left(Y_{n}-Y_{n}^{\prime}>M\right) \geq \mathbb{P}\left(Y_{n}^{+}+\left(Y_{n}^{\prime}\right)^{-}>M, Y_{n}^{-}=\left(Y_{n}^{\prime}\right)^{+}=0\right) \\
& \geq \max \left\{\mathbb{P}\left(Y_{n}^{+}>M,\left(Y_{n}^{\prime}\right)^{+}=0\right), \mathbb{P}\left(\left(Y_{n}^{\prime}\right)^{-}>M, Y_{n}^{-}=0\right)\right\} \\
& \geq \max \left\{\mathbb{P}\left(Y_{n}^{+}>M\right), \mathbb{P}\left(\left(Y_{n}^{\prime}\right)^{-}>M\right)\right\} / 2 .
\end{aligned}
$$

By the tightness condition for $X_{n}-X_{n}^{\prime}$, if we take $M>0$ sufficiently large, the left-hand side can be arbitrary small. This implies the tightnesses of $Y_{n}^{+}$and $Y_{n}^{-}$, and hence the tightness of $Y_{n}=Y_{n}^{+}-Y_{n}^{-}$follows.

\section{REFERENCES}

Akahira, M. and Takeuchi, K. (1995). Non-regular statistical estimation, Number 107 in Lecture notes in statistics, Springer, New York.

Cowles, M. K. and Carlin, B. P. (1996). Markov chain monte carlo convergence diagnostics: A comparative review, J. Amer. Statist. Assoc., 91, 883-904.

Dempster, A. P., Laird, N. M. and Rubin, D. B. (1977). Maximum Likelihood from Incomplete Data via the EM Algorithm, J. R. Stat. Soc. Ser. B, 39(1), 1-38.

Diaconis, P., Khare, K. and Saloff-Coste, L. (2008). Gibbs sampling, exponential families and orthogonal polynomials, Statist. Sci., 23(2), 151-178, ISSN 0883-4237, doi:10.1214/07STS252, with comments and a rejoinder by the authors.

Doob, J. L. (1949). Application of the theory of martingales, Le Calcul des Probabilit es et ses Applications, Colloques Internationaux du Centre National de la Recherche Scienti que, no. 13, pp. 23-27, Centre National de la Recherche Scienti que, Paris.

Gelfand, A. E. and Smith, A. F. M. (1990). Sampling-based approaches to calculating marginal densities, J. Amer. Statist. Assoc., 85(410), 398.

Gilks, W. R., Richardson, S. and Spiegelhalter, D. J., editors (1996). Markov Chain Monte Carlo in Practice, CRC Press.

Hajek, J. (1972). Local asymptotic minimax and admissibility in estimation, Proceedings of the Sixth Berkeley Symposium on Mathematical Statistics and Probability, vol. 1, pp. 175-194.

Hastings, W. K. (1970). Monte Carlo sampling methods using Markov chains and their applications, Biometrika, 57, 97-109.

Imai, K. and van Dyk, D. (2005). A Bayesian analysis of the multinomial probit model using marginal data augmentation, J. Econom., 124(2), 311-334.

Kamatani, K. (2011). Local degeneracy of Markov chain Monte Carlo methods, arXiv:1108. 2477.

Kamatani, K. (2013a). Local weak consistency of Markov chain Monte Carlo methods with application to mixture model, Bull. Inform. Cybern., 45, 103-123.

Kamatani, K. (2013b). Note on asymptotic properties of probit gibbs sampler, RIMS Kokyuroku, 1860, 140-146.

Kamatani, K. (2014). Local consistency of Markov chain Monte Carlo methods, Ann. Inst. Statist. Math., 66(1), 63-74.

Keane, M. (1992). A note on identi cation in the multinomial probit model, J. Bus. Econ. Stat., 193-200.

Lehmann, E. L. (1998). Elements of Large-Sample Theory, Springer Texts in Statistics, Springer, New York.

Lehmann, E. L. and Romano, J. P. (2005). Testing Statistical Hypotheses, Springer, 3rd edition. 
Liu, J. S. and Sabatti, C. (2000). Generalised Gibbs sampler and multigrid Monte Carlo for Bayesian computation, Biometrika, 87(2), 353-369.

Liu, J. S. and Wu, Y. N. (1999). Parameter expansion for data augmentation, J. Amer. Statist. Assoc., 94, 1264-1274.

McCulloch, R., Polson, N. and Rossi, P. (2000). A Bayesian analysis of the multinomial probit model with fully identified parameters, J. Econom., 99, 173-193.

Meng, X.-L. and van Dyk, D. (1999). Seeking efficient data augmentation schemes via conditional and marginal augmentation, Biometrika, 86(2), 301-320.

Metropolis, N., Rosenbluth, W. A., Rosenbluth, M. N., Teller, A. H. and Teller, E. (1953). Equations of state calculations by fast computing machines, Journal of Chemical Physics, 21, 1087-1092.

Omori, Y. (2007). Efficient Gibbs sampler for Bayesian analysis of a sample selection model, Stat. Probab. Lett., 77(12), 1300-1311.

Robert, C. and Casella, G. (2011). A short history of Markov chain Monte Carlo: Subjective recollections from incomplete data, Statist. Sci., 26(1), 102-115, ISSN 0883-4237, doi:10.1214/10-STS351.

Roberts, G. O. and Rosenthal, J. S. (2004). General state space markov chains and mcmc algorithms, Probability Surveys, 1, 20-71.

Roy, V. and Hobert, J. P. (2007). Convergence rates and asymptotic standard errors for Markov chain Monte Carlo algorithms for Bayesian probit regression, J. R. Stat. Soc. Ser. B Stat. Methodol., 69(4), 607-623.

Tanner, M. A. and Wong, W. H. (1987). The calculation of posterior distributions by data augmentation. J. Amer. Statist. Assoc., 82(398), 528-540.

Tierney, L. (1994). Markov chains for exploring posterior distributions, Ann. Statist., 22(4), 1701-1762, ISSN 0090-5364, doi:10.1214/aos/1176325750, with discussion and a rejoinder by the author. 